\title{
P12
}

\section{Dissolution Behaviour of a Binary Mixture in a Capillary Tube}

\author{
M.S.P. Stevar (University of Southampton) \& A. Vorobev (University of \\ Southampton)
}

\section{SUMMARY}

We develop a pore-level physical model for the process of miscible displacement through porous media. Using the network model, the current task is reduced to the study of the dissolution dynamics of a binary mixture within a single capillary tube. Tubes of rather small diameters are considered when the typical diffusion and convective time scales are comparable. The test tube filled with the solute is immersed into the solvent-filled thermostatic bath; no pressure difference between the ends of the tubes is applied. Using a high-resolution video-camera, we study the solvent penetration into the test tube. We examine the evolution of the isobutyric acid/water mixture far from and close to the critical (consolute) point (which is $26 \mathrm{C}$ for this mixture). The mixture fills the circular glass tubes of diameters $0.4 \mathrm{~mm}-0.8 \mathrm{~mm}$ and of various lengths. The shape of the interface and its position are tracked and analysed. Based on our observations the following conclusions can be drawn. In all experiments, we observe a front-type propagation of the solvent phase into the tube with a clearly visible interface. The gravity force significantly affects the shape of the interface and the dissolution dynamics in all undertaken experiments. If the mixture temperature is below the critical point, then the uneven one-sided penetration of the solvent into the tube was consistently observed. The solute/solvent interface experiences oscillations of its shape (being either concave or convex at different time moments). If the mixture temperature is above the critical point, then the solvent penetrates evenly from both ends. In both under- and supercritical conditions, the contact line moves with the same speed as the interface, but the apparent contact angle is time- and coordinate-dependent. The rate of the interface propagation varies at different stages of the dissolution process and does not follow the predictions of the diffusion theory. 


\section{Introduction}

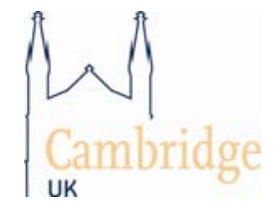

A slow dissolution process of a binary mixture within a porous media is a part of several technologies, e.g. the vegetable oil extraction (Mukhopadhyay 2000), enhanced oil recovery, and enhanced aquifer remediation (Babadagli 2007, Jawitz et al. 1998, and Silva et al. 2005). Basically, these technologies rely on the same physical process, the solvent-based (or miscible) displacement of a solute from the porous medium. The porous media can be roughly represented as a network of interconnected capillary tubes (Aker et al. 1998, Blunt et al. 2002). Such a representation allows us to reduce the initial consideration of this physical process to the study of dissolution within a single capillary tube. The pore sizes are typically small, which makes the hydrodynamic flows within porous media being quite slow, and, as a result, the diffusion is expected to have a significant contribution to the overall mass transport.

In the current work, we undertook a detailed experimental investigation of the dissolution of the solute from a single capillary tube. The aim was to estimate and compare the roles of convection and diffusion in governing the mass transfer in tubes of different diameters and for a binary mixture maintained at various temperatures. We examined the dissolution of the solute by filling the capillary tube, and then immersing the tube with open ends into a solvent-filled thermostatic bath. During the dissolution experiments the tubes were kept in horizontal position. No pressure gradients were imposed between the ends of the tubes. Despite the fact that tubes with rather small diameters were used for the experiments, the gravity significantly affected the dissolution process, which, obviously, could be explained by small surface tension on the solute/solvent interfaces.

The diffusional dissolution of a liquid droplet from the capillary tube was first theoretically examined by Ugrozov et al. (2004). The removal of a small droplet (the size of the droplet was assumed to be larger than the diameter of the tube but much smaller than the tube length) of poorly-dissolving substance was studied. The dissolution was considered as a two-stage process. During the first stage, the solute concentration in the droplet rapidly decreases to the uniform equilibrium value $C_{2}$. This occurs 'fast enough as compared with the diffusion process' or instantaneously in Ugrozov et al. (2004). A tiny fraction of the solute diffuses into the solvent phase and forms the 'concentration tails'. The second stage is explained by the diffusion of the 'concentration tails', modelled as the classical Fickian process. The dissolution rate derived by Ugrozov et al. (2004) is

$$
\frac{d L}{d t}=-\frac{C_{1}}{\sqrt{3-2 C_{1}}} \sqrt{\frac{D}{t}} \approx-\frac{C_{1}}{\sqrt{3}} \sqrt{\frac{D}{t}} .
$$

Here $L$ is the droplet size and $D$ is the constant diffusion coefficient. $C_{1}$ is the equilibrium concentration of the solute in the solvent phase, while $C_{2}$ is the equilibrium concentration of the solute in the solute-rich phase. The solubility, i.e. $C_{1}$, is assumed to be small in Ugrozov et al. (2004).

Formula (1) has not been experimentally verified as even today we are still unaware of any experimental work where the dissolution of a liquid from the capillary tube has been studied.

The dissolution of solid particles surrounded by a solvent were studied, especially, in relation to drugdelivery applications (Wong et al., 1996, Wong et al., 2008, Arifin et al., 2006), and also the dissolution of nonionic surfactants was reported (Chen et al., 2000). The displacement of the solute phase from the capillary tube under applied pressure gradients between the ends of the tube was also extensively studied experimentally and theoretically for miscible and immiscible fluids (Taylor, 1961, Cox, 1962, Petitjeans and Maxworthy, 1996, Chen and Meiburg, 1996, Soares et al., 2005, 2006, Kuang et al., 2003, 2004, Reinelt and Saffman, 1985 and Vanaparthy and Meiburg, 2008).

The focus of the current work is the dissolution rather than the displacement process. We consider the evolution of a miscible system (a binary mixture). The phase diagram of a binary mixture separates the homogeneous (the liquids are completely miscible) and heterogeneous (the liquids are only 
partially miscible) equilibrium states. The components of a heterogeneous binary mixture are separated by an interfacial boundary. In equilibrium, despite the strong difference in concentrations across the interface, the mass transfer through the interface is absent. But the change of the mixture temperature shifts the phase equilibrium and induces the interfacial mass transfer. In addition, the equilibration to a new thermodynamically-stable state can be accompanied by the changes of the interface shape and generation of hydrodynamic flows due to Marangoni (dependence of the surface tension on the solute concentration) or gravity (solutal convection) mechanisms.

The experiments outlined below were set up so that, at the initial moment, the binary mixture was not in its equilibrium state and its evolution to the thermodynamic equilibrium was investigated. The aim of the current work was to consider such regimes in the multiphase liquid/liquid flows when the convective and diffusion time scales could be comparable. Moreover, our first goal was to single out the diffusion process, which, however, was hardly possible to achieve.

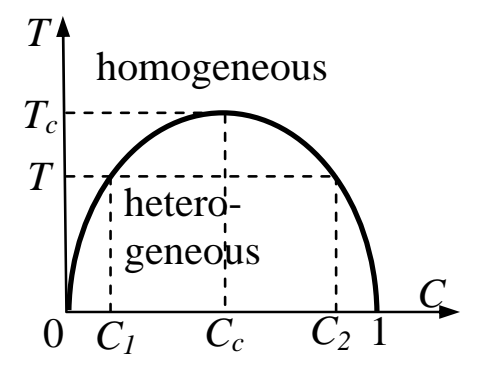

Figure 1. Schematic phase diagram of the isobutyric acid/water binary mixture. The coordinates of the critical point are $T_{c}=299 \mathrm{~K}$ and $C_{c}=0.4$.

The experiments were conducted with the isobutyric acid (IBA)/water binary mixture. This mixture has the phase diagram schematically shown in Figure 1 and has the upper critical solution temperature $T_{c}=299 \mathrm{~K}$.

The phase separation behaviour of the IBA-water mixture was studied experimentally via different methods like light scattering (Chou and Goldburg, 1979, Wong and Knobler, 1981) and more recently by visual observations of the phase separation patterns when temperature gradients were imposed (Seki et al., 2004 and Kinoshita et al., 2005). In these papers, the near-critical behaviour of the diffusion coefficient was determined, but only above the critical point for homogeneous states of the binary mixture. We did not find in literature any measurements of the diffusion coefficient of IBA in water as a function of concentration.

Finally, we can also name some other relevant experimental studies, e.g. the papers of the J. Pojman's group, where the evolution of the miscible droplet in the solvent-filled rotating capillary tube (the configuration of the spinning droplet tensiometry, Pojman et al. 2006) and the evolution of the miscible interface in an immovable cuvette (Viner and Pojman 2008) were investigated. The evolutions of the IBA/water and 1-butanol/water mixtures were studied, with the aims to measure the surface tension of the miscible interface and the rate of diffusion smearing of the interfacial boundary.

\section{Experiment}

In the experiment, the binary mixture filled the borosilicate glass capillary tubes of circular crosssection. Tubes of different diameters $(0.4,0.6$, and $0.8 \mathrm{~mm})$ and lengths (varying from 2 to $15 \mathrm{~cm}$ ) were used. The test tubes were immersed into a transparent water-filled thermostatic bath (with dimensions $100 \times 20 \times 20 \mathrm{~cm}$ ) and were horizontally placed on the stand installed close to the centre of the bath. The temperature of the liquid filling the bath was controlled with a GRANT thermostat (GD100). Several reference points were used to check the homogeneity of the solvent temperature. IBA(99+\%) was purchased from Fisher Scientific. 
The components of the binary mixture were separated by a visible interfacial boundary. Below the critical point the interface was always sharp, while above the critical point the interface was diffuse but remained still visible for a certain period of time. The position and shape of the interfaces were recorded. The images were collected with a system including a CCD camera (LaVision Imager 3S) with Questar lenses (QM100 Model $\ \# 30003$, working distance: $15 \mathrm{~cm}-35 \mathrm{~cm}$ ), a diffuser (LaVision, VZ-illumination high efficiency diffuser) and a diode laser (Oxfordlaser Firefly system). To improve the image quality, the IBA was dyed with a small amount of methylene blue in concentration less than $0.1 \%$. In all snapshots shown below, the darker zone represents the water phase that penetrates into the tube and the pale one represents the IBA-rich phase.

We found that the shape and evolution of the interface were quite complex and could not be explained by simple diffusion not even for the smallest tubes considered (with diameter, $d=0.4 \mathrm{~mm}$ ). In all experiments, the gravity effects were quite substantial over the whole dissolution process.

The observed dissolution behaviour was different for the under- and super-critical temperatures. The first important and not-expected experimental observation was that the penetration occurred through just one end of the tube despite the fact that both ends were in contact with the solvent. Such an uneven one-sided dissolution was observed for all the tubes used (of different diameters and lengths) if the solution temperature was below the critical point. We found that the side of the tube from which the dissolution started was determined by the experimental technique: upon immersion of the test tube into the bath the ends of the tube were manually closed, inevitably, one hand was removed first and the dissolution started from this end of the tube. This was proven by deliberately opening one end after another. The observations also showed that the penetration of the solvent inside the tube from one end was accompanied by the leakage of the solute phase from the opposite end. We also observed that sometimes an end of the tube might become blocked by an air bubble, which stopped the motion of the interface (in this case the diffusion transport became dominant).

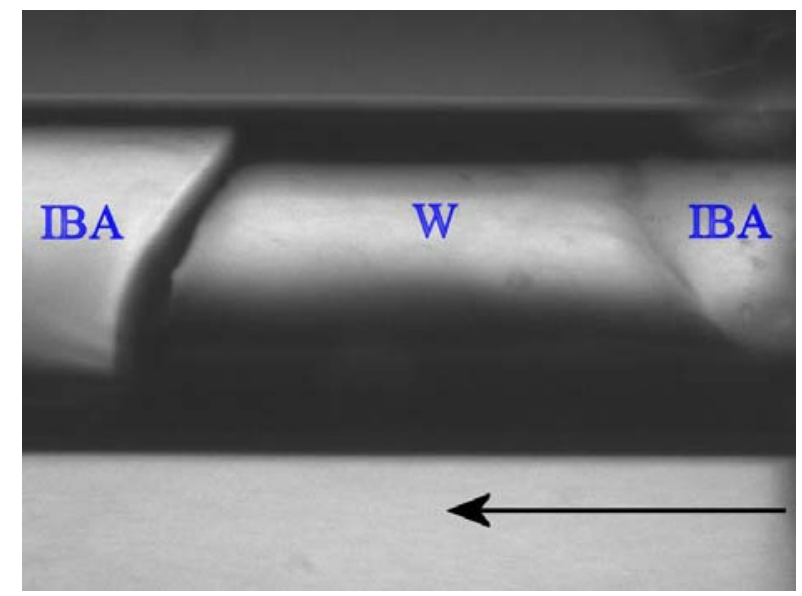

(a)

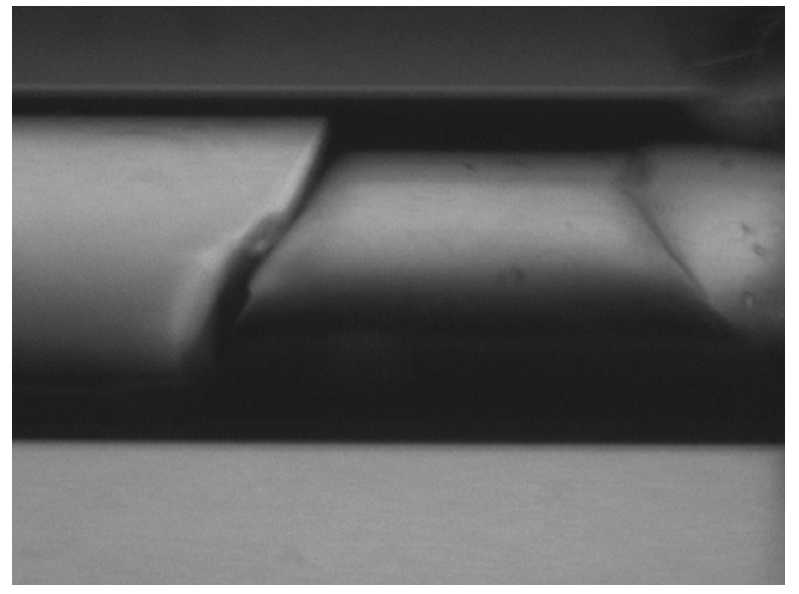

(b) 


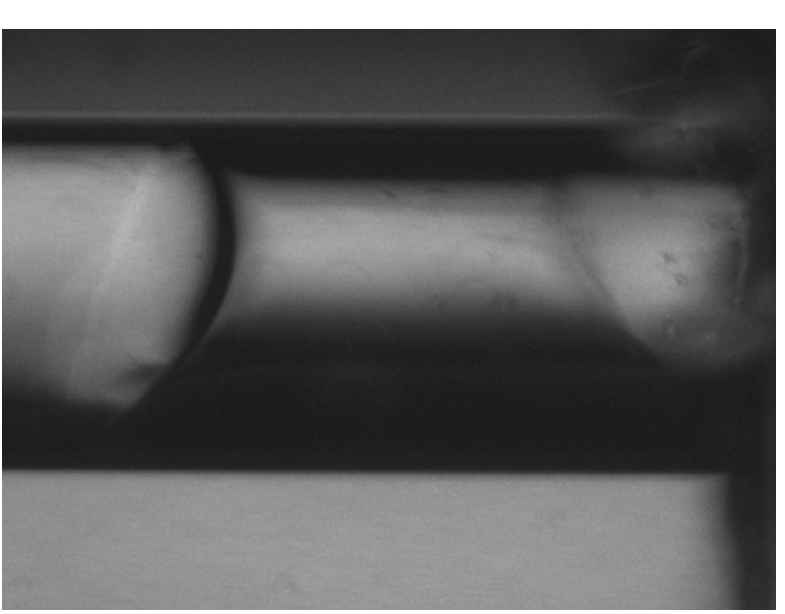

(c)

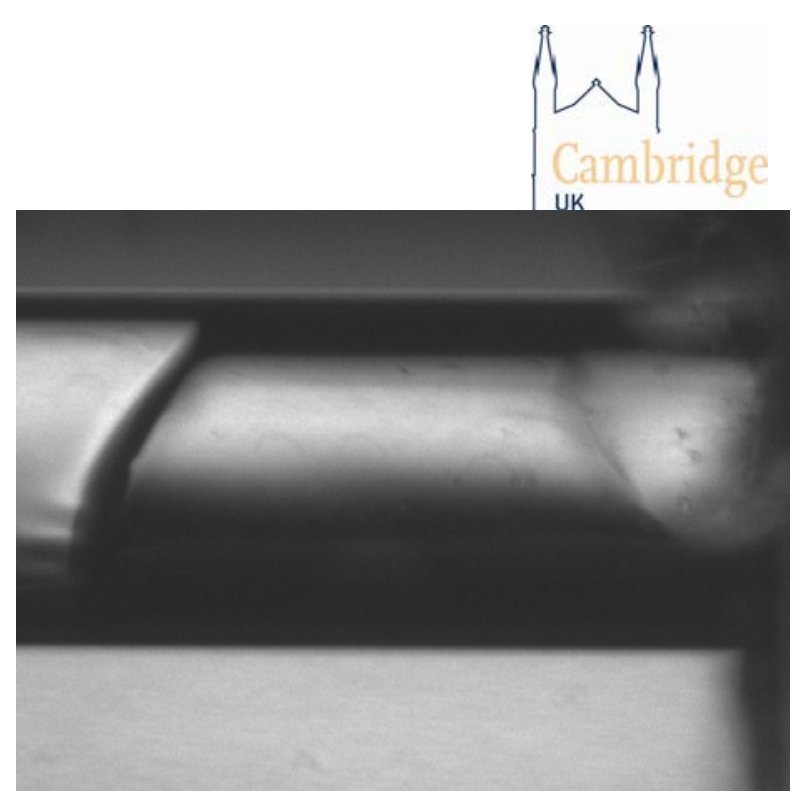

(d)

Figure 2. The entrance moment. The shown interfaces were taken at the time moments $t=43 \mathrm{~s}(a)$, $t=44 \mathrm{~s}(b), t=45 \mathrm{~s}$ (c), and $t=46 \mathrm{~s}(\mathrm{~d})$. The tube diameter was $d=0.8 \mathrm{~mm}$, the length of the tube was $L=10.3 \mathrm{~cm}$ and the mixture temperature was $T=293 \mathrm{~K}$. In (a), the arrow indicates the direction of the interface propagation; the water phase is marked by the letter ' $W$ ' and the isobutyric acid is marked by 'IBA'. The right edge of the image coincides with the right end of the tube. The given time moments are the times from the start of the experiment.

At the time of the entrance, the solute/solvent interface experienced quite complex modifications of its shape. For larger tubes ( $d=0.6 \mathrm{~mm}$ and larger), the initially flat interface became instantly ruptured and the water under-rode the IBA phase forming a droplet of IBA trapped close to the end of the tube. The droplet was later displaced/dissolved, but the newly formed water/solvent interface started propagating inside the tube. This behaviour is shown in Figure 2. In these snapshots, one notices that the water phase is connected with the rest of the bath-water through a channel at the bottom of the tube. It can be also noticed that the left solute/solvent interface experiences oscillations: it can be either convex or concave at different time moments. The outlined entrance effects were more pronounced for the tubes with larger diameters and for the temperatures closer to the critical point when the surface tension becomes even smaller. If the mixture temperature was lower than $298 \mathrm{~K}$ and the tube of the smallest diameter, $d=0.4 \mathrm{~mm}$, was used, then such a behaviour was never observed: the solute/solvent interface remained stable for the whole experiment.

If, however, the mixture temperature was above the critical point then the dissolution scenario was different. Under supercritical temperatures, the solvent evenly penetrated from both ends of the tube. Two interfaces were visible. The interfaces moved towards each other from the opposite ends with almost equal speeds. This is illustrated in Figure 3. 


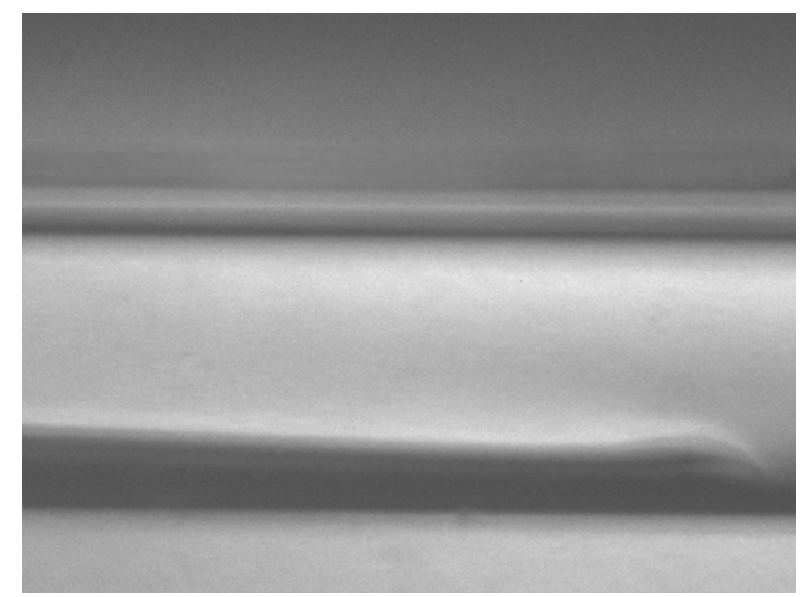

(a)

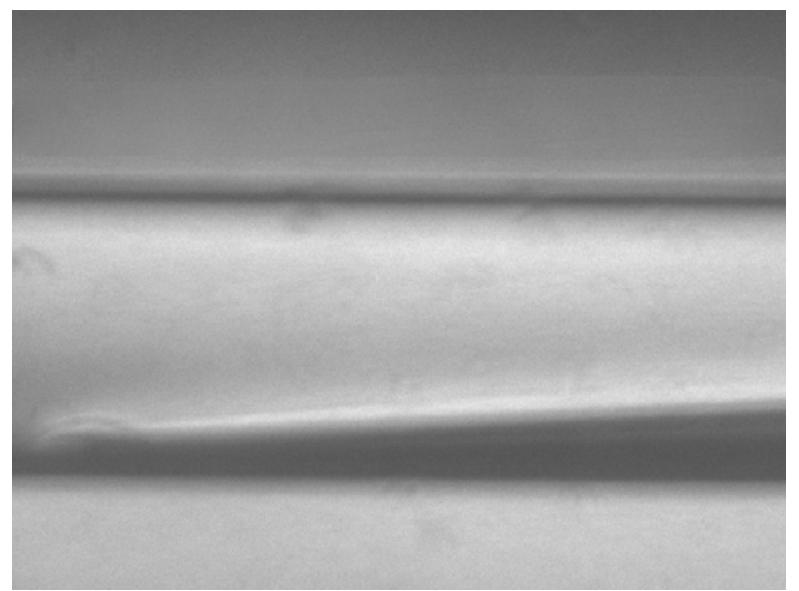

(b)

Figure 3. The entrance moment. The interfaces propagating from the left (a) and right (b) end of the tube are shown. Both interfaces were captured during the same experiment; the left one at the time moment $t=137 \mathrm{~s}$ and the right one at $t=105 \mathrm{~s}$. The tube diameter was $d=0.6 \mathrm{~mm}$, the length of the tube was $L=4 \mathrm{~cm}$ and the mixture temperature was $T=300 \mathrm{~K}$.

When the water/solvent interface penetrated towards the middle of the tube, its shape became stable. The typical snapshots of the already established interfaces are depicted in Figures 4-6. Figure 4 shows the effect of the diameter of the tube: the influence of gravity (namely, an inclination of the interface) was stronger for the tubes of larger diameters. Even, for the smallest tube considered, with the diameter $d=0.4 \mathrm{~mm}$, the interface was still not strictly vertical. The snapshots also show that the contact line moves together with the bulk of the interface; the interface was shifting as a whole without significant changes to its shape. The visible, apparent, contact angle was however different at different time moments and was usually different for the lower and upper ends. The snapshots shown in Figures 4a,b were obtained for the experiments conducted at 293K, but the results obtained at all temperatures up to $298 \mathrm{~K}$ were quite similar. Figures $4 \mathrm{c}$,d show the interfaces for the supercritical temperatures also for the tubes of two different diameters. One sees that the effect of gravity was much stronger in this case. 


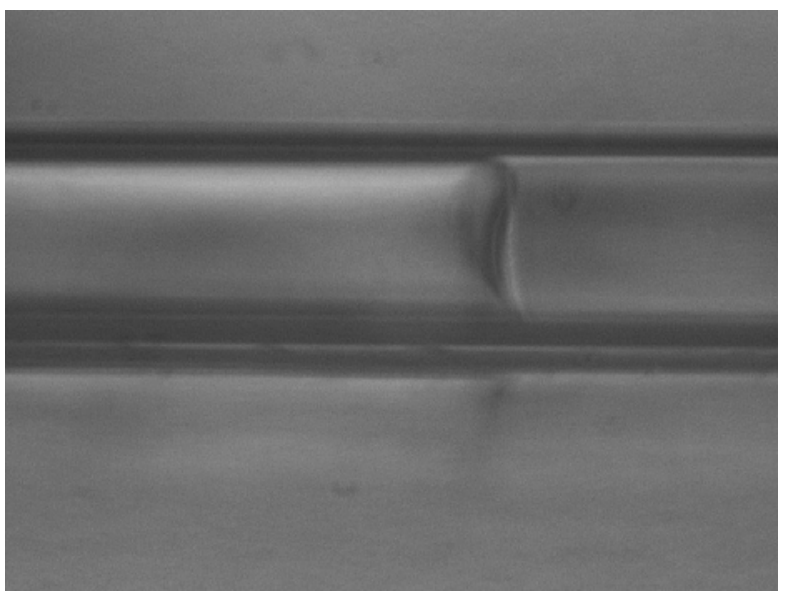

(a)

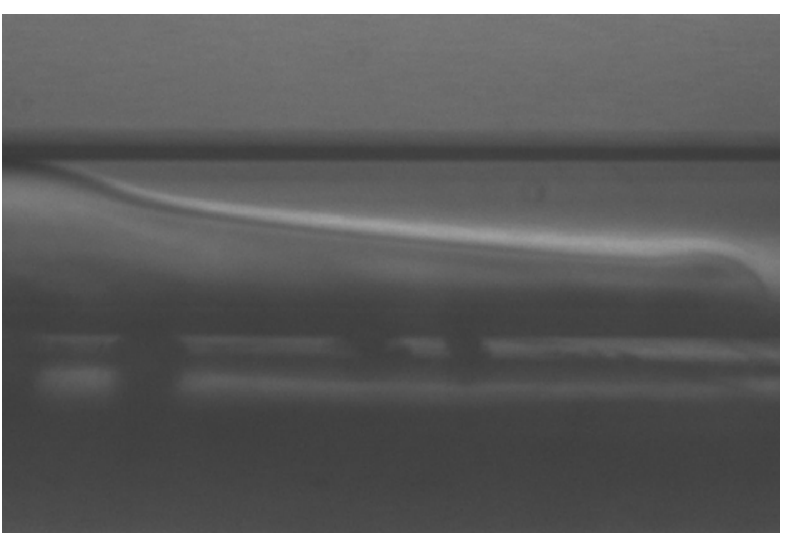

(c)

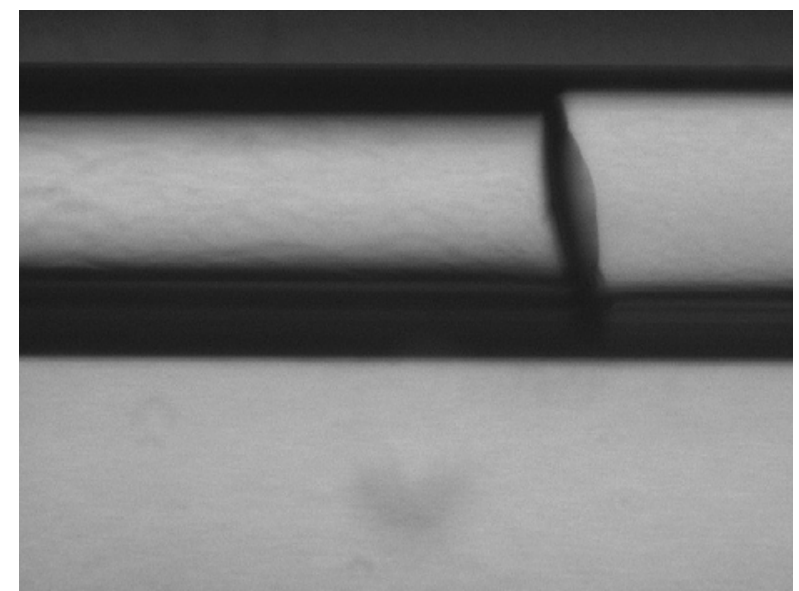

(b)

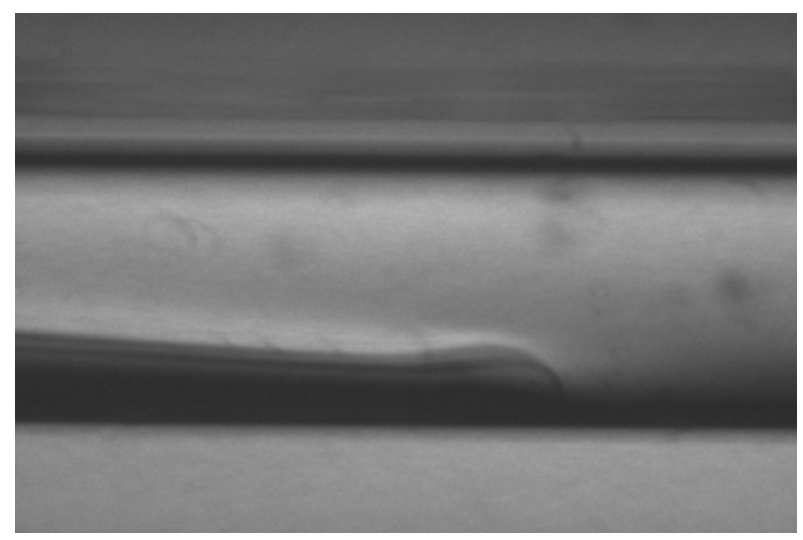

(d)

Figure 4 The shape of the solute/solvent interface for the tubes of different diameters for undercritical $(a-b, T=293 \mathrm{~K})$ and supercritical (c-d, $T=300 \mathrm{~K}$ ) conditions. (a) $d=0.4 \mathrm{~mm}, t=952 \mathrm{~s}$, (b) $d=0.6 \mathrm{~mm}$, $t=387 \mathrm{~s}$, (c) $d=0.4 \mathrm{~mm}, t=76 \mathrm{~s}$, and (d) $d=0.6 \mathrm{~mm}, t=66 \mathrm{~s}$. The snapshots are shown for the steady solute/solvent interfaces established at some distances from the entrance in the tube and remain stable for the rest of the experiment.

Figure 5 shows how the shape of the solute/solvent interface evolved if the mixture temperature was increased. Closer to the critical point, the binary mixture remained heterogeneous, but the surface tension diminished so that the gravity clearly dominated even in the smallest tubes. Above the critical point, the boundary between phases was clearly seen at least for the initial moments of the experiment. As one can see in Figure 5d, the smearing of the interface started from its tip that became hardly visible almost in the beginning of the experiment. Such behaviour could be observed when the mixture temperature was sufficiently high. 


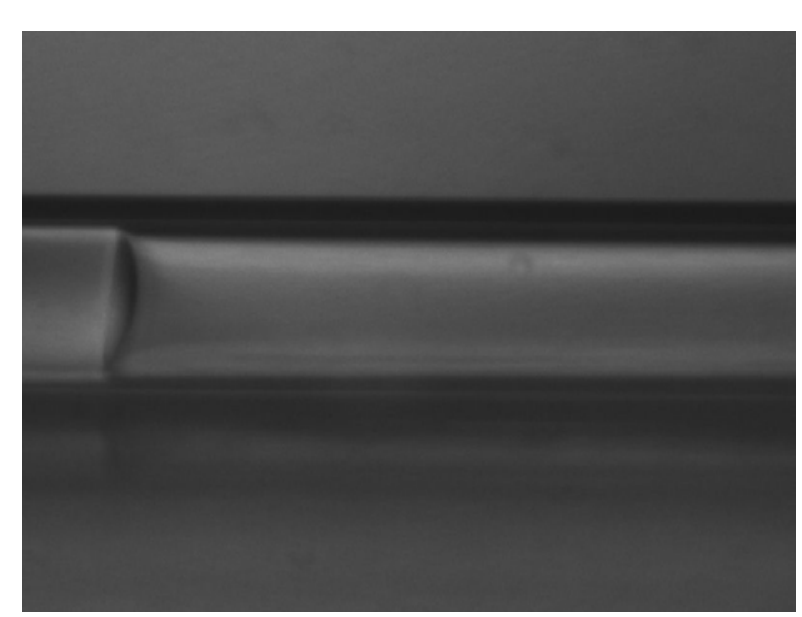

(a)

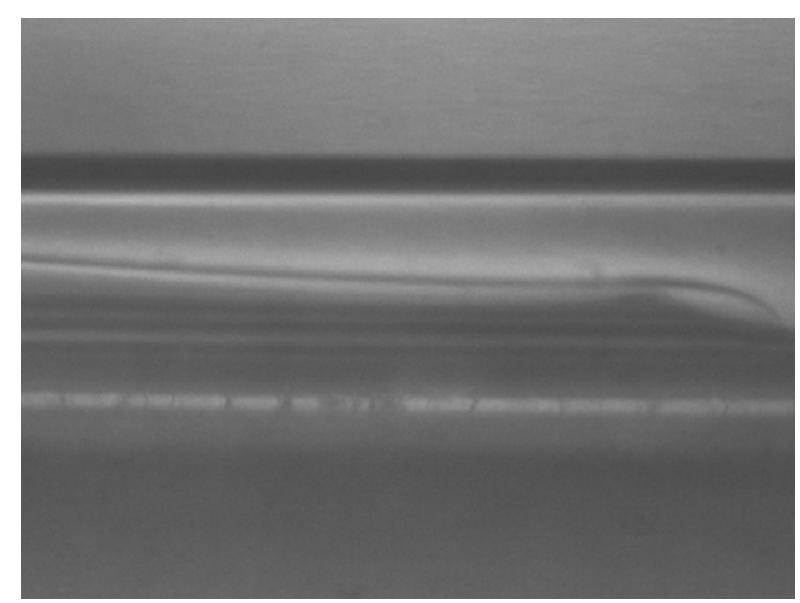

(c)

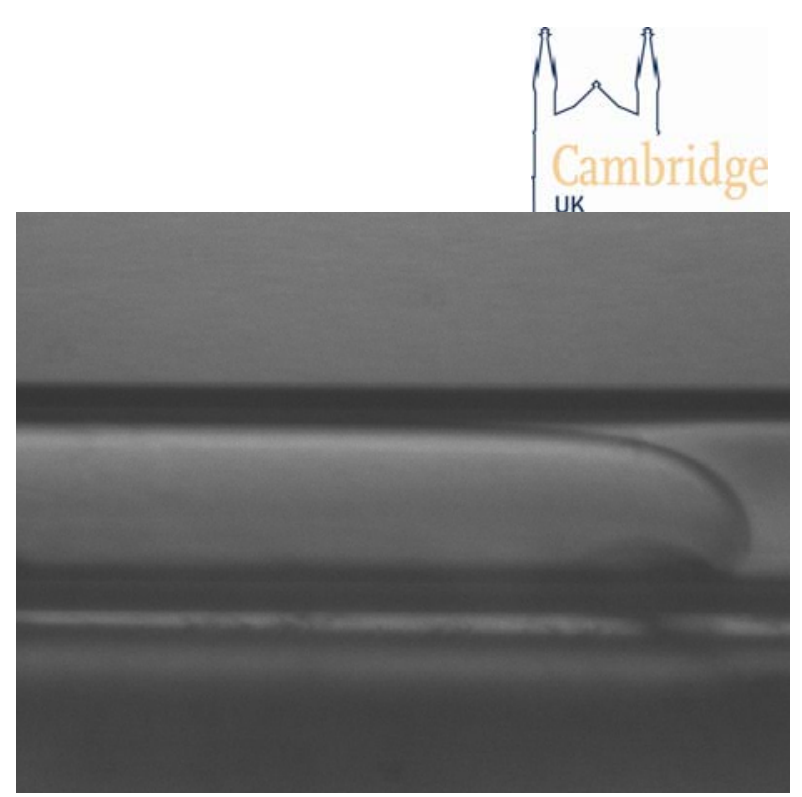

(b)

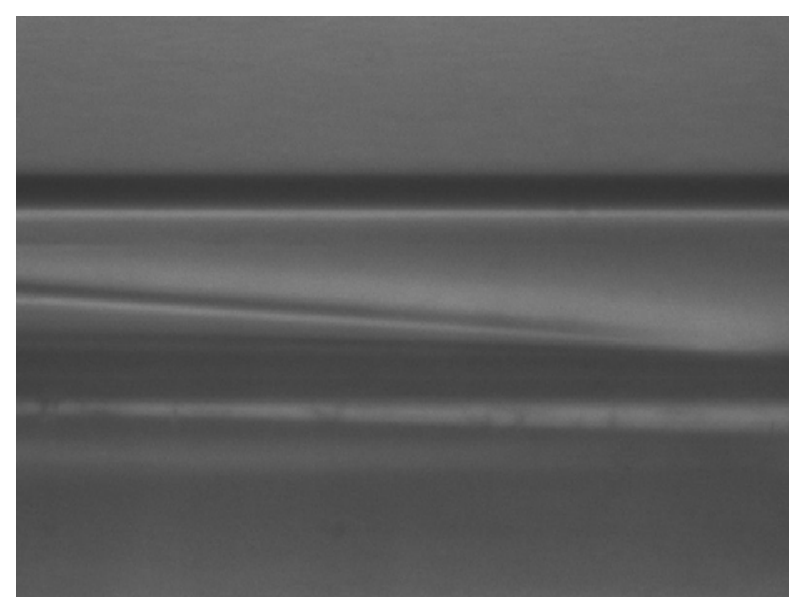

(d)

Figure 5. The shape of the solute/solvent interface for experiments conducted under different temperatures. (a) $T=298 \mathrm{~K}, t=276 \mathrm{~s}$ (b) $T=299 \mathrm{~K}, t=802 \mathrm{~s}$, (c) $T=300 \mathrm{~K}, t=491 \mathrm{~s}$, and (d) $T=303 \mathrm{~K}, t=72$. The tube diameter is the same for all pictures, $d=0.4 \mathrm{~mm}$, the times are the time periods from the beginning of the experiments.

Another interesting observation was that even an established interface always experienced small oscillations as illustrated in Figures 6a-c. These oscillations were observed for the undercritical temperatures. The oscillations were induced by an abrupt start of the dissolution process and could be already seen in Figure 2. The amplitude of oscillations was larger for the tubes of larger diameters and also for the temperatures closer to the critical point. The time-resolution of the visual rig was sufficient only to estimate the period of oscillations that was found to be of order of $1 \mathrm{~s}$. The maximum amplitude of oscillations was observed at the beginning of the dissolution and then the amplitude slowly decayed as the interface moved inside the tube. 

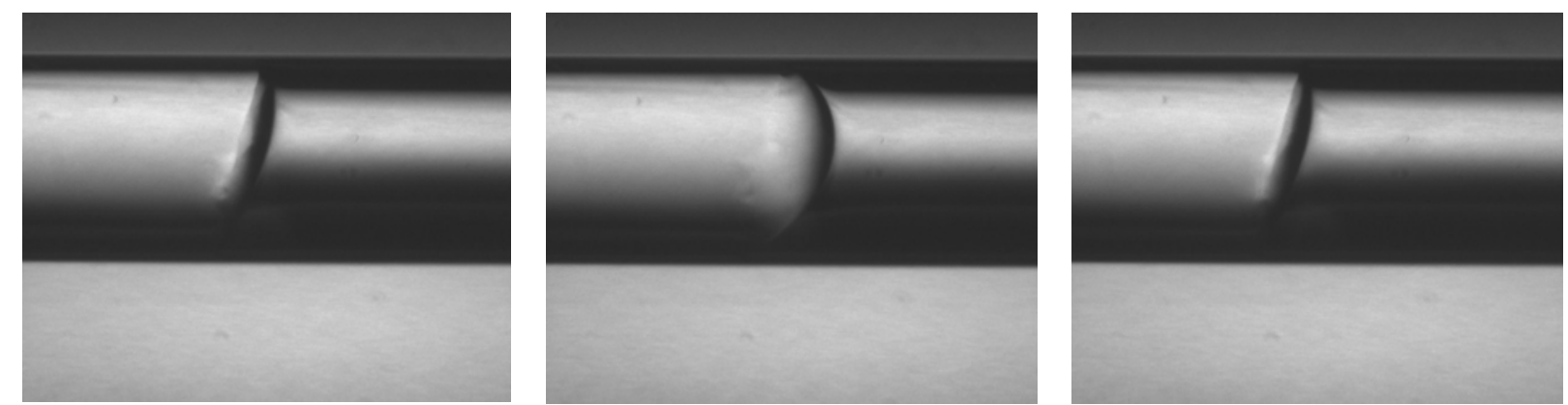

Figure 6. Oscillations of the interface. The sequence of the interfaces for the time moments, $t=621 \mathrm{~s}$, $622 \mathrm{~s}$, and 623s. The diameter of the tube is $d=0.8 \mathrm{~mm}$, the length is $L=10.3 \mathrm{~cm}$ and the temperature is $T=293 K$.

Finally, we measured the rate of propagation of the solute/solvent interface. Since we were able to capture only part of the tube, first, the camera was focused at the end of the tube to snap the video of the interface entrance; when the interface reached the border of the frame, the camera's focus was shifted to follow the interface movement and a new video was captured. This set of videos was analysed and the average velocities were calculated for every position of the camera. The velocities of the interfaces for several experiments are shown in Figure 7.

\section{(a) $d=0.4 \mathrm{~mm}$}

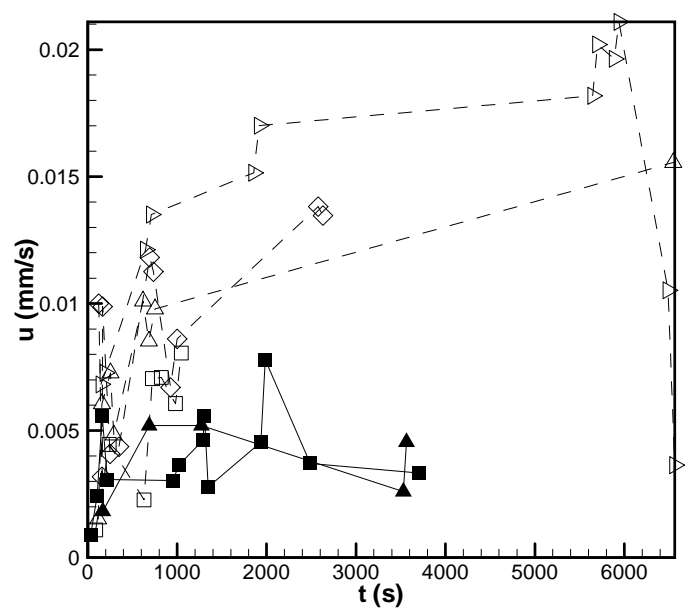

(b) $\mathrm{d}=0.6 \mathrm{~mm}$

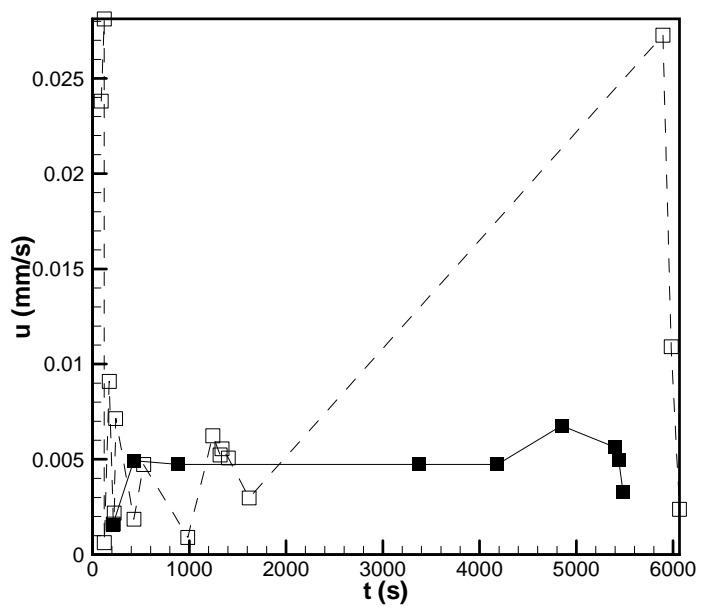

Figure 7. Velocity of the interface. (a) $d=0.4 \mathrm{~mm}, L=4 \mathrm{~cm}$ (solid lines, $T=293 \mathrm{~K}$ and $T=299 \mathrm{~K}$ ) and $L=10.3 \mathrm{~cm}$ (dashed lines, $T=293 \mathrm{~K}, T=295 \mathrm{~K}, T=297 \mathrm{~K}$, and $T=298.5 \mathrm{~K}$ ); (b) $d=0.6 \mathrm{~mm}, L=5 \mathrm{~cm}$, $T=293 K$ (solid line) and $L=10.3 \mathrm{~cm}, T=293 \mathrm{~K}$ (dashed line).

The data shown in Figure 7 allow us to draw only some qualitative conclusions. At the initial moment, the behaviour of the interface is quite irregular, which can be explained by the complex entrance effect. Then, the velocity of the interface propagation increases up to some limiting value and remains approximately constant while the interface is moving through the middle section of the tube. When the interface approaches the opposite end of the tube, its speed seems to drop. Surprisingly, there is no strong dependence of the interface speed on the tube's diameter. The speed of the interface is approximately $5 \cdot 10^{-4} \mathrm{~cm} \cdot \mathrm{s}^{-1}$.

Currently, we obtained only some preliminary dissolution rates for the experiments conducted in the supercritical conditions. These results will be reported later. 


\section{Conclusions}

As stated in the introductory section, the initial aim of the work was to separate the diffusion process. We wanted to find out whether the dissolution can be solely governed by diffusion in capillaries of very small diameters. For micro-systems, the capillary forces are traditionally assumed to dominate. The gravity effects overcome the capillary forces when the typical size is greater than the capillary length, defined as (De Gennes, 2004)

$$
\kappa^{-1}=\sqrt{\frac{\sigma}{\rho g}},
$$

where $\sigma$ is the surface tension coefficient, $\rho$ is the fluid density, but for two-fluid interfaces, this quantity should be replaced by $\varphi \rho_{*}$ with $\varphi$ being the density contrast and $\rho *$ being the average density of the two-fluid system. This length is typically of several mm, and may be even larger for the twofluid systems.

We, however, found that in all undertaken experiments the dissolution was seemingly being gravitydriven. The density contrast of IBA and water is low, $\varphi=0.06$, however, the surface tension of the IBA/water mixture is also quite low, which makes the capillary length being quite small, smaller than $1 \mathrm{~mm}$, for the temperatures relatively far from the critical point, and, obviously, being equal to zero in the vicinity of the critical point.

In undercritical conditions, the solute/solvent interface was almost vertical and does not experience strong transformations over its propagation. The measured dissolution rates do not follow the diffusion time dependence, i.e. $t^{-0.5}$ (see formula (1)). Moreover, the diffusion time that can be calculated on the basis of the measured values of the mutual diffusion coefficient of the IBA/water mixture in the supercritical conditions (Chu, 1968; Kaatze, 2000) are not relevant to the studied process. The measured diffusion coefficient is zero in the critical point; above the critical point, the diffusion coefficient grows according to the formula,

$$
D=D_{0}\left(\frac{T-T_{c}}{T_{c}}\right)^{v} \text {, }
$$

with index $v=0.664$ and amplitude $D_{0}=5.9 \cdot 10^{-10} \mathrm{~m}^{2} \mathrm{~s}^{-1}$. The diffusion time scale, for the tube of $10 \mathrm{~cm}$ length can be estimated as $\tau_{d} \propto 10^{7} \mathrm{~s}$. The typical experimental times of the experiment were much shorter.

The dissolution behaviour was essentially different for the under- and super-critical temperatures. Below the critical point, the interface propagated from one side only. The interface should be endowed with the surface tension and the capillary oscillations of the interface shape were observed. Above the critical point, the front-type propagation of the solvent was observed and the solvent penetrated into the tube from both ends. No oscillations were however observed. Owing to the twosided penetration, the complete dissolution occurred at least twice faster. The contact line usually moved together with the bulk of the interface, however, the contact angle was time and coordinate dependent.

The outlined experimental observations are yet preliminary. Currently, we are developing the theoretical model able to reproduce the experimental results. The strong transformations of the interface shape make the theoretical analysis of such a problem quite difficult. The phase-field (or diffuse-interface) approach seems being most appropriate. The governing equations defining the 
thermo- and hydrodynamic evolution of the binary system were first derived by Lowengrub and Truskinovsky (1998). Owing to dependence of the binary mixture density on the solute concentration, the evolution of two incompressible liquids is defined by the quasi-compressible equations. The full quasi-compressible Cahn-Hilliard-Navier-Stokes equations can be simplified for consideration of the slow dissolution dynamics, when the fast quasi-acoustic processes can be filtered out, similarly to the main idea of the low-Mach number approximation (Paolucci, 1982; Lyubimov et al., 2006a,b). The governing equations for the slow dissolution processes were obtained by Vorobev (2010). The analysis of these equations points out that the concentration gradients are able to induce not only the diffusive flux but also the convective flows by either the Korteweg of buoyancy forces. In addition, the diffusion is governed by the gradient of the chemical potential rather than the gradient of concentration (Landau and Lifshits, 1980) and the expression for the chemical potential includes the barodiffusion and surface tension effects (Lowengrub and Truskinovsky, 1998; Vorobev, 2010).

\section{Acknowledgements}

This work is done within the framework of the EPSRC project EP/G014337 'Pore-Level Network Modelling of the Miscible Displacement.' The authors wish to thank Dr. J. Shrimpton and his students, for helping with the visual rig measurements.

\section{References}

Aker, E., Maloy, K.J., Hansen, A., and Batrouni G. G. [1998] A Two-Dimensional Network Simulator for Two-Phase Flow in Porous Media, Transport in Porous Media, 32, 163-186.

Arifin, D. Y., Lee, L. Y. and Wang, C-H. [2006] Mathematical modeling and simulation of drug release from microspheres: Implications to drug delivery systems, Advanced Drug Delivery Reviews, 58, $1274-1325$.

Babadagli, T. [2007] Development of mature oil fields-A review, Journal of Petroleum Science and Engineering, 57, 221-246.

Blunt, M.J., Jackson, M.D., Piri, M. and Valvatne, P.H. [2002] Detailed physics, predictive capabilities and macroscopic consequences for pore-network models of multiphase flow, Advances in Water Resources, 25, 1069-1089.

Chen, B-H., Miller, C.A., Walsh, J.M., Warren, P.B., Ruddock, J.N., Garrett, P.R., Argoul, F. and Leger, C. [2000] Dissolution rates of pure nonionic surfactants, Langmuir, 16, 5276-5283.

Chen, C.Y. and Meiburg, E. [1996] Miscible displacements in capillary tubes. 2. Numerical simulations, Journal of Fluid Mechanics, 326, 57-90.

Chou, Y.C. and Goldburg, W.I. [1979] Phase separation and coalescence in critically quenched isobutyric-acid-water and 2,6-lutidine-water mixtures, Physical Review, 20(5), 2105-2113.

Chu, B. and Schoenes, F.J. [1968] Diffusion coeffficient of the isobutyric-acid-water system in the critical region, Physical Review Letters, 21, 6-9.

Cox, B.G. [1962] On driving a viscous fluid out of a tube, Journal of Fluid Mechanics,14, 81-96.

De Gennes, P.-G., Brochard-Wyart, F. and Quere, D. [2004] Capillarity and Wetting Phenomena. Drops, Bubbles, Pears, Waves. Springer.

Jawitz, J.W., Annable M.D., and Rao, P.S.C. [1998] Miscible fluid displacement stability in unconfined porous media: Two-dimensional flow experiments and simulations, Journal of Contaminant Hydrology, 31, 211-230. 


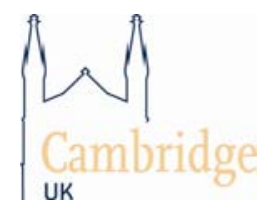

Kaatze, U. and Mirzaev, S.Z. [2000] Slowing down in chemical reactions. The isobutyric acid/water system in the critical region, Journal of Physical Chemistry A, 104, 5430—5436.

Kinoshita, T., Tsukada, T., Hozawa, M., Yokoyama, C. and Kobayashi, M. [2005] Phase separation behavior in a binary mixture fluid layer subjected to vertical temperature and concentration gradients, Chemical Engineering Science, 60, 6939-6946.

Kuang, J., Maxworthy, T. and Petitjeans, P. [2003] Miscible displacements between silicone oils in capillary tubes, European Journal of Mechanics - B/Fluids, 22(3), 271-277.

Kuang, J., Petitjeans, P. and Maxworthy, T. [2004] Velocity fields and streamline patterns of miscible displacements in cylindrical tubes, Experiments in Fluids, 37(2), 301-308.

Landau, L.D. and Lifshitz, E.M. [1980] Statistical Physics, 3rd Edition, Part 1, Pergamon Press.

Lowengrub, J. and Truskinovsky, L. [1998] Quasi-incompressible Cahn-Hilliard fluids and topological transitions, Proceeding of the Royal Society of London, A454, 2617-2654.

Lyubimov, D., Lyubimova, T., Vorobev, A., Mojtabi, A. and Zappoli, B. [2006] Thermal vibrational convection in near-critical fluids. Part 1. Non-uniform heating, Journal of Fluid Mechanics, 564, $159-183$.

Lyubimov, D., Lyubimova, T. Vorobev, A. Mojtabi, A. and Zappoli, B. [2006] Thermal vibrational convection in near-critical fluids. Part 2. Weakly non-uniform heating, Journal of Fluid Mechanics, 564, 185-196.

Mukhopadhyay, M. [2000] Natural extracts using supercritical carbon dioxide, CRC Press LLC.

Paolucci, S. [1982] On the filtering of sound from the Navier-Stokes equations, SAND82-8257, Sandia National Laboratories, Livermore, California.

Petitjeans, P. and Maxworthy, T. [1996] Miscible displacements in capillary tubes. 1. Experiments. Journal of Fluid Mechanics, 326, 37-56.

Pojman, J.A., Whitmore, C., Liveri, M.L.T., Lombardo R., Marszalek, J., Parker, R. and Zoltowski, B. [2006] Evidence for the existence of an effective interfacial tension between miscible fluids: isobutyric acid-water and 1-butanol-water in a spinning-drop tensiometer, Langmuir, 22, 2569—2577.

Reinelt, D.A. and Saffman, P.G. [1985] The penetration of a finger into a viscous fluid in a channel and tube, Siam Journal on Scientific and Statistical Computing, 6(3), 542-561.

Seki, Y. and Okabe, T. and Oshida, Y. and Kobayashi, M. and Tsukada, T. and Hozawa, M. and Kubo, M. [2004] Phase-separation behavior in a binary mixture fluid layer subjected to a vertical temperature gradient, Chemical Engineering Science, 59, 2685-2693.

Silva, A., Delerue-Matos, C., Fiuza, A. [2005] Use of solvent extraction to remediate soils contaminated with hydrocarbons, Journal of Hazardous Materials, 124, 224-229.

Soares, E.J. and Carvalho, M. S. and Souza Mendes, P. R. [2005] Immiscible liquid-liquid displacement in capillary tubes, Journal of Fluids Engineering, 127(1), 24-31.

Soares, E.J.and Carvalho, M.S. and Mendes, P.R.S. [2006] Gas-displacement of non-Newtonian liquids in capillary tubes, International Journal of Heat and Fluid Flow, 27(1), 95-104. 
Taylor, G.I. [1961] Deposition of a viscous fluid on the wall of a tube. Journal of Fluid Mechanics, 10, 161-165.

Ugrozov, V.V., Filipov, A.N., Paraskeva, C.A., Constatinides, G.N. and Starov, V.M. [2004] Diffusive dissolution of a droplet in a capillary, Colloids Surfaces A: Physicochemical and Engineering Aspects, 239, 129-133.

Vanaparthy, S.H. and Meiburg, E. [2008] Variable density and viscosity, miscible displacements in capillary tube, European Journal of Mechanics B-Fluids, 27(3), 268-289.

Viner, G., and Pojman, J.A. [2008] Studying diffusion of partially miscible and systems near their consolute point by laser line deflection, Optics and Lasers in Engineering, 46, 893-899.

Vorobev, A. Boussinesq approximation of the Cahn-Hilliard-Navier-Stokes equations, 2010, Physical Review E, 82, 056312 [10 pages].

Wong, J., Brugger, A., Khare, A., Chaubal, M., Papadopoulos, P., Rabinow, B., Kipp, J. and Ning, J. [2008] Suspensions for intravenous (IV) injection: A review of development, preclinical and clinical aspects, Advanced Drug Delivery Reviews, 60, 939-954.

Wong, J., Kuu, W-Y., Kajimoto, H. and Kazama, H. [1996] Correlation of the intravenous in-line drug delivery kinetics with the diluent flow rate, angle of internal flow, wettability, solubility and particle surface area, International Journal of Pharmaceutics, 144, 177-186.

Wong, N-C. and Knobler, C.M. [1981] Light-scattering studies of phase separation in isobutyric acid+water mixtures: Hydrodynamic effects, Physical Review A, 24(6), 3205-3211. 